\title{
Philosophiques
}

\section{Introduction : Littérature et connaissance}

\section{Pascal Engel}

Volume 40, numéro 1, printemps 2013

Littérature et connaissance

URI : https://id.erudit.org/iderudit/1018372ar

DOI : https://doi.org/10.7202/1018372ar

Aller au sommaire du numéro

Éditeur(s)

Société de philosophie du Québec

ISSN

0316-2923 (imprimé)

1492-1391 (numérique)

Découvrir la revue

Citer ce document

Engel, P. (2013). Introduction : Littérature et connaissance. Philosophiques, 40(1), 3-7. https://doi.org/10.7202/1018372ar d'utilisation que vous pouvez consulter en ligne.

https://apropos.erudit.org/fr/usagers/politique-dutilisation/ 


\title{
Introduction: Littérature et connaissance
}

\author{
PASCAL ENGEL
}

(UNIGE/ EHESS)

On entend dire plus souvent que par le passé que la littérature a quelque chose à voir avec la vérité et avec la connaissance, et qu'elle n'est pas ce monde clos sur lui-même, purement langagier, sans lien avec la moindre réalité, que dénonça jadis Julien Benda sous le nom de littérature "pure» et «byzantine ${ }^{1}$. Ceux-là mêmes qui l'avaient adorée nous disent que cette conception met la littérature en péril'2. Ils redécouvrent le sens commun, l'esthétique du vraisemblable, et retrouvent dans le roman des ressources de pensée que son statut prétendu de pure fiction avait éclipsées. Mais de quelle réalité la littérature ainsi redécouverte est-elle la connaissance et de quelle vérité s'agit-il ? De la réalité sociale, comme à la grande époque du naturalisme? De la réalité historique? De la réalité morale? De la vie humaine en général ? Certes, mais comment? En quel sens des mots «connaissance» et du mot "vérité »? Va-t-on revenir à Taine, à Zola, à Paul Bourget? Ou bien aux conceptions épiphaniques de la vérité des romantiques de l'Athenaeum? Le problème ne se pose plus dans les mêmes termes que quand on avait seulement le choix entre une conception purement formaliste et autoréférentielle de la littérature et une conception "humaniste». Nombre de travaux sur le roman, la fiction, et la connaissance littéraire ont modifié le paysage ${ }^{3}$. Les essais réunis dans ce volume de Philosophiques en prennent acte.

Une bonne voie d'entrée dans ces questions est ce que l'on appelle «le paradoxe de la fiction ", qui n'est en fait qu'une généralisation d'une remarque d'Aristote dans la Poétique: nous prenons plaisir, et éprouvons de la terreur et de la pitié face aux tragédies qui contiennent une représentation (mimésis) de la réalité, quand bien même nous voyons que ce qui se passe sur scène n'est pas la réalitét. Le paradoxe consiste dans le fait que les trois énoncés suivants semblent tous vrais, alors qu'ils se contredisent:

(I) Nous éprouvons terreur et pitié au théâtre;

(II) Mais pour éprouver terreur et pitié face à quelque chose, il faut la croire réelle;

(III) Nous croyons que les faits représentés au théâtre ne sont pas réels.

1. Julien Benda, La France byzantine, Paris, Gallimard, 1945.

2. Tzvetan Todorov, La littérature en péril, Paris, Flammarion, 2007.

3. Cf. notamment Peter Lamarque \& Stein Olsen, Truth Fiction and Literature, Oxford, Oxford University Press, Martha Nussbaum, Love's Knowledge, Oxford, Oxford University Press, trad. fr. La connaissance de l'amour, Paris, Cerf, 2010; Jacques Bouveresse, La connaissance de l'écrivain, Marseille, Agone 2010; Thomas Pavel, La pensée du roman, Paris Gallimard.

4. Voir William Marx, «La véritable catharsis aristotélicienne», pour une lecture philologique et physiologique de la Poétique, Poétique, 20 I I-2, n I66, p. I3 I-I 54. 
Il y a, de prime abord, trois types de solutions possibles à ce paradoxe, qui fait l'objet d'au moins deux des contributions de ce volume, celles de Maurizio Ferraris et celle de Carola Barbero. On peut en premier lieu rejeter l'idée que nous éprouvions des émotions authentiques en assistant à des représentations théâtrales ou en lisant des œuvres de fiction: peut-être n'éprouvonsnous que des quasi-émotions, ou des émotions feintes, et peut-être faisons-nous seulement semblant de croire que ce qui nous est représenté est réel. On peut en second lieu rejeter l'idée qu'éprouver des émotions face à des œuvres de fiction entraîne que l'on croie à la réalité des entités décrites dans celles-ci. Les conceptions dites "cognitives" des émotions supposent que celles-ci sont associées à un contenu de croyance à propos d'un certain objet ou état de chose (par exemple pour avoir peur du chien on doit croire qu'il est dangereux), mais d'autres conceptions soutiennent que les émotions reposent sur des affects ou des comportements qui ne sont pas associés essentiellement à des représentations ${ }^{5}$. Si l'émotion ne suppose aucune croyance en la réalité de ce qui est représenté, le paradoxe disparaît. Enfin, la troisième proposition est plus difficile à rejeter que les autres, car comment évaluer une œuvre de fiction sans d'une manière ou d'une autre ne pas croire que la référence et la vérité y sont suspendues ou absentes?

Le paradoxe de la fiction sert de révélateur pour la question de l'existence et de la nature de la connaissance littéraire. Si l'on remplace, dans la formulation précédente du paradoxe de la fiction, «éprouver terreur et pitié » par «éprouver des émotions », et «au théâtre » par «en lisant des fictions", on obtient le paradoxe de la fiction tel qu'il est discuté aujourd'hui. Peut-on généraliser ce paradoxe en l'étendant aux œuvres littéraires en général ?

(i) Les œuvres littéraires nous procurent des émotions.

(ii) Mais pour avoir des émotions, il faut croire que ce qui nous émeut est réel.

(iii) Nous savons que la littérature ne donne aucune connaissance du réel.

C'est moins clair. D'abord parce que la notion de «littérature », à la différence de celle de fiction, est normative et non pas descriptive. Nous ne savons pas toujours bien ce qu'est une fiction (par rapport - par exemple - à un article de journal, un essai, une chronique, un témoignage historique, a fortiori un reportage romancé ou une satire), mais nous savons encore moins si une œuvre est littéraire ou pas, parce que tout est ici affaire de jugements de valeur, et de ce que nous attendons de la littérature, dont l'histoire montre qu'elle a correspondu, selon les époques et les lieux, à des attentes très variées. Qu'est-ce qui est littéraire ou qu'est-ce qui ne l'est pas? Nous jugeons que l'œuvre de J. M. Coetzee relève de la littérature et pas celle de Barbara Cartland, et nous trouvons les œuvres de Réjean Ducharme plus lit-

5. Voir, pour une introduction récente, Julien Deonna et Fabrice Teroni, Qu'est-ce qu'une émotion? Paris, Vrin, 2008, trad. angl. The Emotions, London, Routledge, $20 \mathrm{I} 2$. 
téraires que celles de Denise Bombardier, mais quelle est exactement la différence?

Si l'on se limite à la notion de fiction, le paradoxe sert encore de prisme pour poser le problème de la connaissance littéraire parce qu'il met en jeu deux idées communes: l'une que les fictions, en particulier littéraires, ne relèvent pas du discours vériconditionnel, susceptible de vérité ou de fausseté, et l'autre que les fictions littéraires suscitent en nous, lecteurs, certaines émotions. La première idée est indispensable si l'on veut pouvoir soutenir en un sens qui ne soit pas purement métaphorique qu'il y a une connaissance littéraire, car qui dit connaissance dit vérité: si l'on sait que $p, p$ ne peut qu'être vrai. Le paradigme de la connaissance est la connaissance propositionnelle de vérités. Il y a, bien entendu, des conceptions différentes de la vérité, comme celle selon laquelle celle-ci consiste en une forme de révélation, passant par quelque expérience mystique. On peut aussi défendre l'idée que la vérité est une forme de cohérence, auquel cas le roman répond aisément à l'appel, puisque nombre de fictions romanesques sont cohérentes, et en ce sens vraies. Tant qu'on ne dit pas en quoi la cohérence pourrait entraîner la vérité, cette conception ne nous dit pas grand-chose. Il y a aussi des conceptions plus faibles de la connaissance, comme celle selon laquelle il suffit, pour qu'il y ait connaissance, que le discours en question ait un "pouvoir inférentiel ", ou qu'il ait "une valeur cognitive ", suscitant en nous des idées, peut-être à l'aide de l'imagination. On dit souvent que les expériences de pensée en ce sens ont une valeur cognitive, c'est-à-dire sont des auxiliaires à la connaissance, sans apporter à proprement parler de connaissance. La fiction littéraire et romanesque serait connaissance en ce sens affaibli, parce que les propositions qu'on y trouve ne peuvent pas être littéralement vraies. Si c'est le cas, alors la troisième prémisse du paradoxe de la fiction est fausse. C'est ce que soutient une conception que l'on peut appeler «expressiviste» de la connaissance littéraire, par analogie avec la théorie en méta-éthique qui veut que nos énoncés moraux ne soient que des projections de nos attitudes et ne renvoient à aucune réalité morale. De même la littérature de fiction aurait seulement un pouvoir expressif, projetant nos sentiments, au contact de l'œuvre, sur des réalités qui n'en sont pas. C'est en fait la conception que soutiennent deux des auteurs les plus discutés dans la tradition récente, Peter Lamarque, et Stein Olsen, dans leur livre Truth, Fiction and Literature (I994), qui fait ici en partie l'objet des contributions de Jean Marie Schaeffer et de Stelios Virvidakis. Selon la conception "pas-de-vérité" de Larmaque et Olsen, la vérité littérale, et donc la connaissance littérale sont absente de l'œuvre littéraire, qui peut au mieux exprimer des états mentaux, des attitudes, dans des contextes, le plus souvent sociaux et culturels, et en réponse à des préoccupations le plus souvent éthiques (en quoi cette conception se rattache à la conception dite "humaniste" de la littérature). Dans sa contribution, Maurizio Ferraris défend aussi une version de la thèse expressiviste, en soutenant que la thèse selon laquelle nous n'éprouvons, vis-à-vis des fictions, 


\section{Philosophiques / Printemps 2013}

que des quasi-émotions, est fausse. Selon Ferraris, c'est la prémisse selon laquelle nos croyances au sujet des entités fictives sont vraies ou fausses qu'il faut rejeter: de même que bien des discours de la vie courante n'ont pas pour fonction d'énoncer des jugements vrais ou faux, la littérature n'a pas cette fonction. Une autre manière de soutenir la conception expressiviste présente dans la contribution de Jean-Marie Schaeffer est de proposer l'idée que la connaissance littéraire est une forme de connaissance pratique, au sens de l'acquisition indirecte d'un savoir-faire, distincte par nature d'un savoir théorique et propositionnel. C'est une idée séduisante, mais elle appelle une théorie du savoir pratique. Si celui-ci est censé ne pas avoir affaire à la vérité, mais au faire, la notion de connaissance prend un sens très différent. Si on veut pouvoir effectivement défendre l'idée de connaissance littéraire, il est impossible de se ranger à la conception expressiviste.

Une autre manière d'envisager cette connaissance consiste à examiner la notion de fiction elle-même. C'est la voie qu'a empruntée la majeure partie de la critique littéraire récente. Outre celle de Schaeffer, deux des contributions réunies ici abordent le problème sous cet angle, mais de manière assez différente des nombreux travaux qui ont mis l'accent sur la sémantique des énoncés fictionnels. Carola Barbero soutient la thèse, d'inspiration meinongienne, selon laquelle dans la fiction nous ne sommes pas mis en relation avec des objets de référence (possibles ou réels), mais avec des propriétés, ce qui lui permet de suspendre la troisième prémisse du paradoxe de la fiction. Alexis Tadié s'interroge sur la conception de fiction à partir d'une étude historique sur la pensée de Bacon, qui se demandait, avec ses contemporains: où commence l'histoire, où commence le récit, où commence la fiction? Il montre que les penseurs et écrivains anglais de l'âge classique - en particulier Defoe - avaient une conscience aiguë de ces questions et une manière subtile de les aborder.

Prenons à présent le paradoxe du côté de la question de la nature des émotions, et principalement de celles que sont censées produire sur nous les œuvres littéraires. Quelles sont les sortes d'émotions que nous procurent les œuvres littéraires? Il semble que cela ait à voir avec l'échelle de valeurs que nous accordons aux œuvres. Les ouvrages dont l'unique objectif est de faire pleurer Margot ou de faire rire Basile sont jugés de peu de valeur, alors que ceux qui élèvent l'âme, le sens moral ou le sens esthétique nous semblent mériter mieux de la littérature. Selon de nombreuses conceptions des émotions, celles-ci sont la source ou la manifestation des valeurs, morales, esthétiques ou autres. Mais quelle que soit la nature exacte de la relation entre les émotions et les valeurs, il nous faudra une conception de la nature de ces valeurs si nous voulons pouvoir soutenir que la connaissance littéraire est, directement ou non, connaissance de valeurs. Il faudra d'abord déterminer de quelles valeurs il peut s'agir. Selon bien des conceptions défendues aujourd'hui, 
la littérature nous donne une forme de connaissance morale ${ }^{6}$. Mais pourquoi faudrait-il que la connaissance littéraire se limite à celle-ci et aux valeurs éthiques seulement? Des auteurs aussi différents que Stendhal, Musil et Miłosz, analysés ici respectivement par Patrizia Lombardo et par Kevin Mulligan, soutiennent que les valeurs qui concernent avant tout l'écrivain sont les valeurs intellectuelles, les valeurs qui précisément ont rapport avec une bonne sensibilité à la connaissance et à la vérité ${ }^{7}$. C'est la raison pour laquelle, suggère ici Mulligan, bien des écrivains sont obsédés par le problème de la bêtise, qui est un défaut de sensibilité au vrai et au savoir en général. Et, comme le montre ici Lombardo au sujet de Stendhal, la connaissance littéraire peut être une connaissance de vérités essentielles non seulement sur la morale conception que je serais volontiers tenté de caractériser comme victorienne et d'appliquer, à l'instar de Nussbaum, surtout à des œuvres anglophones comme celle de George Eliot ou d'Henry James - mais aussi sur la nature humaine et sur les émotions humaines elles-mêmes. Dans ma propre contribution, je suggère que si la conception propositionnelle et la conception expressiviste de la connaissance littéraire sont fausses, elles pourraient toutes deux être vraies en partie si l'on concevait la connaissance pratique non pas, comme on le fait le plus souvent, en opposition à la connaissance propositionnelle, mais comme une forme particulière de connaissance propositionnelle.

Les travaux réunis ici proviennent en partie d'un colloque tenu à Genève en 2009, avec l'aide du FNS (projet "Connaissance, raisons et normes») et de l'Université de Genève. Je remercie Kevin Mulligan et Alexis Tadié d'avoir accepté de me confier leurs essais, et Davide Fassio pour son aide pour la traduction du texte de Maurizio Ferraris, Aude Bandini pour sa traduction du texte de Carola Barbero, un lecteur et une relectrice anonyme pour leurs remarques et Christian Nadeau pour son accueil et son soutien.

6. Voir les livres cités plus haut, de Jacques Bouveresse, de Martha Nussbaum, et, pour une excellente mise au point sur les relations entre art et morale en général, C. Talon Hugon, Morales de l'art, Paris, PUF, 2009.

7. Un autre auteur qui a vu cela est Benda. Je me permets de renvoyer à P. Engel, Les lois de l'esprit, Paris, Ithaque, $20 \mathrm{I} 2$. 